\title{
M2-branes Coupled to Antisymmetric Fluxes
}

\author{
Miao Li], Tower Wang 2 ] \\ Interdisciplinary Center for Theoretical Study, \\ University of Science and Technology of China, \\ Hefei, Anhui 230026, China \\ Institute of Theoretical Physics, Chinese Academy of Sciences, \\ P. O. Box 2735 Beijing 100080, China
}

\begin{abstract}
By turning on antisymmetric background fluxes, we study how multiple M2-branes are coupled to them. Our investigation concentrates on the gauge invariance conditions for the Myers-Chern-Simons action. Furthermore, the dimensional reduction of M2-branes to D2-branes introduces more constraints on the newly introduced tensors. Particularly, for the theory based on $\mathcal{A}_{4}$ algebra, we are able to fix all components of them up to an overall normalization constant. These results can not be simply obtained from the previously proposed cubic matrix representation for this algebra. We also comment on cubic matrices as the representations of 3-algebras.
\end{abstract}

\footnotetext{
${ }^{1}$ E-mail: mli@itp.ac.cn

${ }^{2}$ E-mail: wangtao218@itp.ac.cn
} 


\section{Introduction}

The dynamics of D-branes was well studied a decade ago [1, 2, 3, 4, 5], which has driven huge progress of string theory. In contrast, multiple M-branes are more untamable. Recently, a 3-dimensional field theory for multiple M2-branes was proposed by Bagger and Lambert [6, 7, 8] and Gustavsson [9, 10]. To check this theory, it is important to do the dimensional reduction from M2-branes to D2-branes. The reduction has been investigated from different viewpoints in [9, 11, 12, 22]. By virtue of the Nambu-Poisson algebra, Ho and Matsuo et.al. have carefully worked over the relations between multiple M2-brans and a M5-brane [13, 14, 15, 22].

The construction of Bagger-Lambert-Gustavsson model relies on a 3-algebra with a positive-definite metric, namely the $\mathcal{A}_{4}$ algebra. It was conjectured by [13] and confirmed by [16, 17] that the BLG theory is unique [10, 18], because all finite dimensional 3-algebras with a positive-definite metric are direct sums of $\mathcal{A}_{4}$ with trivial algebras [13, 17]. However, if we do not require the metric to be positive-definite, there are still a rich class of models [19]. In particular, very recently, a class of models based on 3 -algebras with a Lorentzian metrid 3 were studied by several groups [20, 21, 22]. Besides considering Lorentzian metrics, various attempts to extending the BLG model were also made during the past few months [12, 13, 23, 24].

In 25, 26, 27, there are some interesting discussions relating BLG theory to M2branes on an obifold (or namely an "M-fold" [26]). As a partial list, other aspects of BLG theory were extensively studied in [30, 31, 32, 33, 34, 35, 36, 37.

Our interest in this paper is to consider the effects of the background antisymmetric tensor fields on multiple M2-branes. It is well-known that in the world-volume theory of D-branes, when the background fluxes are switched on, there will be additional Chern-Simons terms [2, 3], through which the background fields couple not only to the internal gauge fields but also to the world-volume scalar fields [5]. In string theory, the antisymmetric tensor fields are sourced by various dimensional D-branes or strings. In M-theory, these are 3-forms $C_{(3)}$ and 6-forms $C_{(6)}$, they are dual to each other and are coupled to M2-branes and M5-branes respectively. The Chern-Simons terms we want to discuss describe interactions between world-volume fields and $C_{(3)}$, $C_{(6)}$. To distinguish them from those Chern-Simons terms purely of internal gauge fields (that is, the terms appearing in Bagger and Lambert's papers [7, 8]), we will call them Myers-Chern-Simons terms. Since M2-branes can be obtained by lifting

\footnotetext{
${ }^{3}$ The constructions in [20, 21, 22] are more general, which lead to a 3-algebra with a Lorentzian metric if one starts with a compact semi-simple Lie 2-algebra as a special case.
} 
D2-branes in string theory, it is important to understand these terms for multiple M2-branes.

First of all, we suggest that to lowest order, the Myers-Chern-Simons action for multiple M2-branes takes the form

$$
\begin{aligned}
\mathcal{S}_{M C S}= & \int d^{3} \sigma\left[\lambda_{1} \epsilon^{\lambda \mu \nu} C_{I J K} \operatorname{STr}\left(T^{a} T^{b} T^{c}\right) D_{\lambda} X_{a}^{I} D_{\mu} X_{b}^{J} D_{\nu} X_{c}^{K}\right. \\
& \left.+\lambda_{2} \epsilon^{\lambda \mu \nu} C_{I J K L M N} \operatorname{STr}\left(\left[T^{d}, T^{e}, T^{f}\right] T^{a} T^{b} T^{c}\right) X_{d}^{I} X_{e}^{J} X_{f}^{K} D_{\lambda} X_{a}^{L} D_{\mu} X_{b}^{M} D_{\nu} X_{c}^{N}\right] .
\end{aligned}
$$

When writing down this action, we only take the lowest order terms into consideration, although it is expectable that a full Myers-Chern-Simons action will involve higher order terms of $C_{(3)}, C_{(6)}$ and $A_{\lambda a b}$ and their derivatives. Here $\epsilon^{\lambda \mu \nu}(\lambda, \mu, \nu=1,2,3)$ is the Levi-Civita symbol with $\epsilon^{123}=1$ along the world-volume directions. The coefficients $\lambda_{1}$ and $\lambda_{2}$ depend on conventions, so we leave them as unfixed parameters at present. The covariant derivative with respect to the internal gauge field $A_{\lambda a b}$ is [7, 8]

$$
D_{\lambda} X_{a}^{I}=\partial_{\lambda} X_{a}^{I}-A_{\lambda d c} f_{a}^{d c b} X_{b}^{I}
$$

In this paper, we always use "STr" to denote the symmetrized trace as they did in [4, 5], e.g. (not one appearing in (10)

$$
\begin{aligned}
& \operatorname{STr}\left(\left[T^{a}, T^{b}, T^{c}\right] T^{d} T^{e}\right) \\
= & \frac{1}{3 !}\left[\operatorname{Tr}\left(\left[T^{a}, T^{b}, T^{c}\right] T^{d} T^{e}\right)+\operatorname{Tr}\left(T^{d} T^{e}\left[T^{a}, T^{b}, T^{c}\right]\right)+\operatorname{Tr}\left(T^{e}\left[T^{a}, T^{b}, T^{c}\right] T^{d}\right)\right. \\
& \left.+\operatorname{Tr}\left(\left[T^{a}, T^{b}, T^{c}\right] T^{e} T^{d}\right)+\operatorname{Tr}\left(T^{e} T^{d}\left[T^{a}, T^{b}, T^{c}\right]\right)+\operatorname{Tr}\left(T^{d}\left[T^{a}, T^{b}, T^{c}\right] T^{e}\right)\right],
\end{aligned}
$$

and "Tr" to denote an ordinary trace. But, in the present case of multiple M2branes, we do not know how to perform such a trace "Tr" unless one gives the correct representation of all generators $\left\{T^{a}\right\}$. To circumambulate this difficulty, let us introduce some succinct notations

$$
\begin{aligned}
g^{a b c} & =g^{(a b c)}=\mathrm{S} \operatorname{Tr}\left(T^{a} T^{b} T^{c}\right), \\
d^{a b c d} & =d^{(a b c d)}=\mathrm{S} \operatorname{Tr}\left(T^{a} T^{b} T^{c} T^{d}\right) .
\end{aligned}
$$

The tensors $g^{a b c}$ and $d^{a b c d}$ will be very useful. They are completely symmetric under the permutation of indices as indicated. In our conventions, indices inside round brackets $\left(a_{1}, \ldots, a_{n}\right)$ will always be understood as symmetrized with unit weight, i.e. with a factor $1 / n$ !. We will use the same unit weight convention to anti-symmetrize indices inside square brackets $\left[a_{1}, \ldots, a_{n}\right]$. 
Then in terms of notations (44), the lowest order Myers-Chern-Simons action becomes

$$
\begin{aligned}
\mathcal{S}_{M C S}= & \int d^{3} \sigma\left[\lambda_{1} \epsilon^{\lambda \mu \nu} C_{I J K} g^{a b c} D_{\lambda} X_{a}^{I} D_{\mu} X_{b}^{J} D_{\nu} X_{c}^{K}\right. \\
& \left.+\lambda_{2} \epsilon^{\lambda \mu \nu} C_{I J K L M N} d^{g a b c} f^{d e f}{ }_{g} X_{d}^{I} X_{e}^{J} X_{f}^{K} D_{\lambda} X_{a}^{L} D_{\mu} X_{b}^{M} D_{\nu} X_{c}^{N}\right] .
\end{aligned}
$$

This form of action may be still correct when background fields $C_{(3)}, C_{(6)}$ are functionals of non-Abelian scalars $X^{I}, X^{J}$, etc. But in our following investigation we will consider constant background fields for simplicity. Furthermore, to systematically neglect other terms induced by the metric of spacetime, we work in a flat spacetime background.

The remaining of this paper is organized as follows. In section 2 we derive the general conditions of gauge invariance for the above Myers-Chern-Simons action. After a brief review of Lie 2-algebras in section 3, we solve the gauge invariance conditions and the constraints arising from dimensional reduction, hence fix almost all of the components appearing in (4). This is done in concrete examples, i.e., section 4 for $\mathcal{A}_{4}$, and section 5 for 3 -algebras with a Lorentzian metric. Failing to reobtain the above results in terms of cubic matrices, in section 6, we present some tentative thoughts on cubic matrices as representations of 3-algebras. Section 7 is a short conclusion.

\section{Gauge Invariance}

One of the most apparent constraints on action (5) comes from the requirement that it should be gauge invariant. Under the gauge transformation

$$
\delta X_{a}^{I}=\Lambda_{f e} f_{a}^{f e d} X_{d}^{I}, \quad \delta\left(D_{\lambda} X_{a}^{I}\right)=\Lambda_{f e} f_{a}^{f e d} D_{\lambda} X_{d}^{I},
$$

the first part of (5) changes as

$$
\begin{aligned}
& \delta\left[\epsilon^{\lambda \mu \nu} C_{I J K} g^{a b c} D_{\lambda} X_{a}^{I} D_{\mu} X_{b}^{J} D_{\nu} X_{c}^{K}\right] \\
= & \epsilon^{\lambda \mu \nu} C_{I J K} \Lambda_{f e}\left(g^{d b c} f_{d}^{f e a}+g^{d a c} f_{d}^{f e b}+g^{d a b} f_{d}^{f e c}{ }_{d} D_{\lambda} X_{a}^{I} D_{\mu} X_{b}^{J} D_{\nu} X_{c}^{K},\right.
\end{aligned}
$$

while the gauge transformation of the second part gives

$$
\begin{aligned}
& \delta\left[\epsilon^{\lambda \mu \nu} C_{I J K L M N} d^{g a b c} f^{d e f}{ }_{g} X_{d}^{I} X_{e}^{J} X_{f}^{K} D_{\lambda} X_{a}^{L} D_{\mu} X_{b}^{M} D_{\nu} X_{c}^{N}\right] \\
= & \epsilon^{\lambda \mu \nu} C_{I J K L M N} \Lambda_{j i}\left[d^{g a b c}\left(f^{j i d}{ }_{h}^{e f h}{ }_{g}-f^{j i e}{ }_{h} f_{g}^{d f h}+f^{j i f}{ }_{h} f^{d e h}{ }_{g}\right)\right. \\
& \left.+\left(d^{g h b c} f_{h}^{j i a}+d^{g h a c} f^{j i b}{ }_{h}+d^{g h a b} f^{j i c}{ }_{h}\right) f^{d e f}{ }_{g}\right] X_{d}^{I} X_{e}^{J} X_{f}^{K} D_{\lambda} X_{a}^{L} D_{\mu} X_{b}^{M} D_{\nu} X_{c}^{N} .
\end{aligned}
$$


Here we only consider constant background fields. In general $C_{(3)}, C_{(6)}$ are functionals of non-Abelian scalars $X^{I}, X^{J}$, etc, then more terms will be involved in (7) and (8).

According to (7) and (8), the gauge invariance of the Myers-Chern-Simons terms (5) imposes the conditions

$$
\begin{aligned}
g^{d(a b} f^{c) f e} & =0, \\
d^{g a b c} f_{h}^{j i[d} f_{g}^{e f] h}+d^{g h(a b} f^{c) j i}{ }_{h}^{d e f}{ }_{g} & =0 .
\end{aligned}
$$

This system of equations appears to be over-determined because there are much more equations than independent unknowns. However, these equations are not independent of each other. Obviously they are trivially satisfied for

$$
g^{a b c}=0, \quad d^{a b c d}=0 .
$$

Of course this is not the solution we want. We shall see in all concrete examples, the gauge invariance conditions (9) allow for non-trivial solutions. With the help of computer, given the structure constants $f_{d}^{a b c}$ of an algebra, we can solve numerous linear equations (9) by brute-force, then get the simplified conditions for $g^{a b c}$ and $d^{a b c d}$. This is exactly what we will do in sections 4 and 5. Before doing that, to establish our conventions and notations, we briefly collect some well-known facts about Lie 2-algebras in section 3 ,

\section{Lie 2-Algebras}

In string theory, a $U(N)$ internal gauge symmetry is present on $N$ coincident Dbranes, reducing to $S U(N)$ when we do not consider the center-of-mass (or zero) mode. As concrete examples, we start with $U(2)$ and $U(3)$.

\section{1 $S U(2)$ and $U(2)$}

The $S U(2)$ algebra is well-known,

$$
\left[T^{a}, T^{b}\right]=f_{c}^{a b} T^{c}
$$

where $a, b, c=1,2,3$, and the structure constants $f^{a b}{ }_{c}=f \epsilon^{a b c}$ with $\epsilon^{123}=1$. The representation (planar) matrices of its generators are proportional to the Pauli matrices,

$$
T^{1}=\frac{f}{2}\left(\begin{array}{cc}
0 & i \\
i & 0
\end{array}\right), \quad T^{2}=\frac{f}{2}\left(\begin{array}{cc}
0 & 1 \\
-1 & 0
\end{array}\right), \quad T^{3}=\frac{f}{2}\left(\begin{array}{cc}
i & 0 \\
0 & -i
\end{array}\right) .
$$


$S U(2)$ can be enlarged to $U(2)$ by appending a $U(1)$ factor and the corresponding generator

$$
T^{0}=\frac{f}{2}\left(\begin{array}{ll}
i & 0 \\
0 & i
\end{array}\right)
$$

As a consequence,

$$
\left[T^{0}, T^{0}\right]=\left[T^{0}, T^{a}\right]=0, \quad\left[T^{a}, T^{b}\right]=f^{a b}{ }_{c} T^{c},
$$

with $a, b, c=1,2,3$ and $f^{a b}{ }_{c}=f \epsilon^{a b c}$.

Indices $0, a, b$ and so on are raised by the metric

$$
\begin{aligned}
& h^{00}=\operatorname{Tr}\left(T^{0}, T^{0}\right)=h \propto f^{2}, \\
& h^{0 a}=h^{a 0}=\operatorname{Tr}\left(T^{a}, T^{0}\right)=0, \\
& h^{a b}=\operatorname{Tr}\left(T^{a}, T^{b}\right)=h \delta^{a b} \propto f^{2} \delta^{a b} .
\end{aligned}
$$

In our notations, $f$ determines the normalization of structure constants, and $h$ is the normalization constant for the metric. Please notice that the normalization of the metric and the structure constants should be chosen properly. Throughout this paper, for Lie 2-algebras, we normalize $h^{a b}=\delta^{a b}$ by fixing $h=1$, and keep the normalization constant $f$ here in the structure constants.

\section{$3.2 S U(3)$ and $U(3)$}

The $U(3)$ algebra is described by (14), but with $a, b, c=1,2,3, \ldots 8$, and the structure constants

$$
\begin{aligned}
& f^{a b c}=f^{[a b c]}, \quad f^{123}=f, \\
& f^{147}=-f^{156}=f^{246}=f^{257}=f^{345}=-f^{367}=\frac{f}{2}, \\
& f^{458}=f^{678}=\frac{\sqrt{3}}{2} f .
\end{aligned}
$$


In the planar matrix representation, its generators are proportional to Gell-Mann matrices and the identity matrix,

$$
\begin{aligned}
& T^{1}=\frac{f}{2}\left(\begin{array}{lll}
0 & i & 0 \\
i & 0 & 0 \\
0 & 0 & 0
\end{array}\right), \quad T^{2}=\frac{f}{2}\left(\begin{array}{ccc}
0 & 1 & 0 \\
-1 & 0 & 0 \\
0 & 0 & 0
\end{array}\right), \quad T^{3}=\frac{f}{2}\left(\begin{array}{ccc}
i & 0 & 0 \\
0 & -i & 0 \\
0 & 0 & 0
\end{array}\right), \\
& T^{4}=\frac{f}{2}\left(\begin{array}{lll}
0 & 0 & i \\
0 & 0 & 0 \\
i & 0 & 0
\end{array}\right), \quad T^{5}=\frac{f}{2}\left(\begin{array}{ccc}
0 & 0 & 1 \\
0 & 0 & 0 \\
-1 & 0 & 0
\end{array}\right), \quad T^{6}=\frac{f}{2}\left(\begin{array}{ccc}
0 & 0 & 0 \\
0 & 0 & i \\
0 & i & 0
\end{array}\right), \\
& T^{7}=\frac{f}{2}\left(\begin{array}{ccc}
0 & 0 & 0 \\
0 & 0 & 1 \\
0 & -1 & 0
\end{array}\right), T^{8}=\frac{f}{2 \sqrt{3}}\left(\begin{array}{ccc}
i & 0 & 0 \\
0 & i & 0 \\
0 & 0 & -2 i
\end{array}\right), T^{0}=\frac{f}{\sqrt{6}}\left(\begin{array}{ccc}
i & 0 & 0 \\
0 & i & 0 \\
0 & 0 & i
\end{array}\right) .
\end{aligned}
$$

Getting rid of the $U(1)$ part and hence $T^{0}$, one immediately obtains the algebra $S U(3)=U(3) / U(1)$, which is dictated by (11) and (16).

\section{3-Algebras with a Euclidean Metric}

In [6, 7, 8, 9, 10], a 3-algebra

$$
\left[T^{a}, T^{b}, T^{c}\right]=f_{d}^{a b c} T^{d}, \quad(a, b, c, d=1,2,3,4)
$$

named $\mathcal{A}_{4}$ algebra, is constructed to describe the dynamics of multiple M2-branes. The metric of this algebra is positive-definite, with the signature $(+,+,+,+)$. In a suitable basis, its metric can be chosen as $h^{a b}=\delta^{a b}$. Interestingly, one can obtain $\mathcal{A}_{4}$ by lifting the $S U(2)$ algebra (11) with the new generator $T^{4}$. That is

$$
\begin{aligned}
& {\left[T^{4}, T^{a}, T^{b}\right]=f_{c}^{4 a b}{ }_{c} T^{c}=-f_{c}^{a b}{ }_{c}^{c},} \\
& {\left[T^{a}, T^{b}, T^{c}\right]=f^{a b c}{ }_{4} T^{4}=f^{a b c} T^{4} . \quad(a, b, c, d=1,2,3)}
\end{aligned}
$$

This algebra can be easily extended by considering the center-of-mass mode,

$$
\left[T^{0}, T^{a}, T^{b}\right]=0 . \quad(a, b=1,2,3,4)
$$




\section{$4.1 \quad$ Lifted $S U(2)$}

As we have just shown, in the absence of the center-of-mass mode, $\mathcal{A}_{4}$ can be obtained by lifting $S U(2)$ with a generator $T^{4}$. Its structure constants are given by

$$
f_{d}^{a b c}=f^{[a b c d]}=f \epsilon^{a b c d}, \quad(a, b, c, d=1,2,3,4)
$$

Using these structure constants to solve the gauge invariance conditions (9), we find most components of the symmetrized traces vanish, except for

$$
d^{1111}=d^{a a a a}=3 d^{a a b b}, \quad(a, b=1,2,3,4, \quad a \neq b)
$$

and those components obtained by reordering their indices.

\section{2 $\quad$ Lifted $U(2)$}

If we include the center-of-mass mode and the corresponding generator $T^{0}$, then the $S U(2)$ group is enlarged to $U(2)$. In this way, the $\mathcal{A}_{4}$ algebra is also augmented as in (20), resulting in a direct sum of an $\mathcal{A}_{4}$ with a $U(1)$ algebra. The structure constants are totally anti-symmetric under the exchange of indices, and

$$
\begin{aligned}
& f_{d}^{a b c}=f^{a b c d}=f \epsilon^{a b c d}, \\
& f^{0 a b}=-f^{a b c}{ }_{0}=f^{0 a b c}=0 . \quad(a, b, c, d=1,2,3,4)
\end{aligned}
$$

Making use of them to solve (9), we find the other components of the symmetrized traces should vanish, except for the following components

$$
\begin{aligned}
& g^{000}, \quad g^{011}=g^{0 a a}, \quad d^{0000}, \quad d^{0011}=d^{00 a a}, \\
& d^{1111}=d^{a a a a}=3 d^{a a b b}, \quad(a, b=1,2,3,4, \quad a \neq b)
\end{aligned}
$$

and those obtained by reordering their indices, such as $g^{101}, g^{110}$ etc. That is to say, the gauge invariance allows for non-trivial solutions besides the trivial solution (10).

\subsection{Reduction to D2-branes}

The above results in (22) and (24) can be readily understood through the gauge theory on two coincident D2-branes. Even the undetermined components can be fixed by reduction to D2-branes.

Taking an appropriate normalization for $\lambda_{1}$ and $\lambda_{2}$, we can perform the ordinary trace for any planar matrix $T$ as usual, by summing over the diagonal elements,

$$
\operatorname{Tr}(T)=\sum_{i}(T)_{i i}
$$


In this way, substituting (12) and (13) directly into definitions (41), one quickly get

$$
\begin{aligned}
& g^{000}=g^{011}=g^{022}=g^{033}=-\frac{i f^{3}}{4}, \\
& d^{0000}=d^{0011}=d^{0022}=d^{0033}=\frac{f^{4}}{8}, \\
& d^{1111}=d^{2222}=d^{3333}=\frac{f^{4}}{8}, \\
& d^{1122}=d^{1133}=d^{2233}=\frac{f^{4}}{24},
\end{aligned}
$$

and

$$
g^{012}=0, \quad g^{123}=0, \quad d^{0012}=0, \quad d^{1112}=0, \quad d^{1123}=0,
$$

and so on.

The 3-algebras in BLG theory naturally arise from a non-associative product, so it is difficult to imagine that their generators can be represented by planar matrices. Indeed, Ho et. al. have emphasized in [13, 38] that cubic matrices are a more suitable representation for 3-algebras [39, 40]. But if we take the cubic matrix representation proposed in [13], it seems impossible to recover the above results.

For a single cubic matrix $T$ with three indices, the only natural definition of a trace is the summation over diagonal elements [40],

$$
\operatorname{Tr}(T)=\sum_{i}(T)_{i i i}
$$

This definition is a trivial extrapolation of (25), and can be trivially extrapolated to "general matrices" with even more indices [40]. In [13] a cubic matix representation for $\mathcal{A}_{4}$ generators was proposed

$$
\left(T^{a}\right)_{i j k}=\left|\epsilon^{a i j k}\right| \exp \left(\frac{i \pi}{8} \epsilon^{a i j k}\right), \quad(a, i, j, k=1,2,3,4)
$$

which meets well the algebra (18) under a triple product defined as in [13, 39],

$$
(A, B, C)_{i j k}=\sum_{l} A_{l i j} C_{l j k} B_{l k i}
$$

But unfortunately, using these rules and this representation to calculate (4), we simply get

$$
g^{000}=0, \quad g^{011}=g^{022}=g^{033}=g^{044}=0,
$$

which are at odds with (26). We will return to this point in section 6.

Now let us go back to see why the calculations (26) and (27) make sense. 
Following the strategy invented in [11, we can perform the dimensional reduction for the Myers-Chern-Simons action (5). To get more details please refer to subsection 5.6 and references [11, 20, 22]. The resultant action contains the required MyersChern-Simons terms for multiple D2-branes and a high-dimensional term,

$$
\begin{aligned}
\mathcal{S}= & \int d^{3} \sigma\left(\mathcal{L}_{M C S}^{D 2}+\mathcal{L}_{H D}\right) \\
\mathcal{L}_{M C S}^{D 2}= & \frac{\lambda_{1}}{g_{Y M}^{3}} \epsilon^{\lambda \mu \nu} C_{I J K} g^{a b c} D_{\lambda} X_{a}^{I} D_{\mu} X_{b}^{J} D_{\nu} X_{c}^{K} \\
& -\frac{\lambda_{2}}{g_{Y M}^{4}} \epsilon^{\lambda \mu \nu} C_{8 I J L M N} d^{g a b c} f_{g}^{d e} X_{d}^{I} X_{e}^{J} D_{\lambda} X_{a}^{L} D_{\mu} X_{b}^{M} D_{\nu} X_{c}^{N}, \\
\mathcal{L}_{H D}= & -\frac{3 \lambda_{1}}{g_{Y M}^{3}} C_{8 I J} g^{a b c} F_{a}^{\mu \nu} D_{\mu} X_{b}^{I} D_{\nu} X_{c}^{J}
\end{aligned}
$$

with $I, J, \ldots=1,2,3, \ldots, 7$, and $a, b, \ldots=0,1,2,3$. We have neglected terms involving the goldstones and $X_{\phi}^{I}$. In principle goldstones should be eaten after a redefinition of fields. It is remarkable that the high-dimensional term $\mathcal{L}_{H D}$ is new. This term is hard to see from the world-volume theory of D2-branes, but easy to obtain via dimensional reduction of M2-branes.

Because the $U(2)$ gauge group induced by D2-branes can be represented by planar matrices (12) and (13), in this situation, it is reasonable to utilize the planar matrices to calculate the tensors $g^{a b c}$ and $d^{a b c d}$ with indices $a, b, c, d=0,1,2,3$. Since the action (32) is reduced from (5), these components should take the same values in them. Therefore the results (26) and (27) can be trusted, even though $\mathcal{A}_{4}$ may not be represented by planar matrices.

Can we also determine the values of components with the index 4? Observing that in $\mathcal{A}_{4}$ algebra there is a symmetry between $T^{1}, T^{2}, T^{3}$ and $T^{4}$, we can get these components simply by replacing index 1 with index 4 , or replacing index 2 or 3 with index 4. This observation helps us deduce the other components by virtue of (26) and (27),

$$
g^{044}=-\frac{i f^{3}}{4}, \quad d^{0044}=\frac{f^{4}}{8}, \quad d^{4444}=\frac{f^{4}}{8}, \quad d^{1144}=\frac{f^{4}}{24}, \quad \ldots
$$

Hence we have proved solution (24) and fixed the non-vanishing components.

\section{3-Algebras with a Lorentzian Metric}

Although $\mathcal{A}_{4}$ successfully incorporates the $S U(2)$ algebra, its generalization to $S U(N)$ for an arbitrary $N$ turns out to be very difficult. If one requires 
1. there is an invariant positive-definite metric,

2. the structure constants are totally anti-symmetric and satisfy the fundamental identity,

3. the 3-algebra is finite, non-trivial and irreducible,

then $\mathcal{A}_{4}$ algebra is the unique 3 -algebra [10, 18, 13, 16, 17]. Such a "uniqueness theorem" can be bypassed in many ways via relaxing some of the above requirements. Very recently, permitting at least one negative signature in the metric, three groups [20, 21, 22] independently introduced another way to lift any Lie 2-algebra $\mathcal{G}$ to a 3-algebra.

For arbitrary Lie 2-algebra

$$
\left[T^{a}, T^{b}\right]=f_{c}^{a b} T^{c}
$$

by introducing two new generators $T^{+}$and $T^{-}$, they lifted it to a 3 -algebra [20, 21, 22]

$$
\begin{aligned}
{\left[T^{-}, T^{a}, T^{b}\right] } & =0, \\
{\left[T^{+}, T^{a}, T^{b}\right] } & =f^{+a b}{ }_{c} T^{c}=f^{a b}{ }_{c} T^{c}, \\
{\left[T^{a}, T^{b}, T^{c}\right] } & =f^{a b c}{ }_{-} T^{-}=f^{a b c} T^{-} .
\end{aligned}
$$

When $\mathcal{G}$ is positive-definite, the metric of such a 3 -algebra is not positive-definite, but has a Lorentzian signature $(-,+,+, \ldots+)$. Being interested in Myers-ChernSimons action for this class of algebras, we will take $\mathcal{G}$ to be $S U(N)$ or $U(N)$ and study some concrete examples. In a suitable basis, the metric of a lifted $U(N)$ algebra is of the form $[20,21,22$.

$$
h^{+-}=-1, \quad h^{ \pm \pm}=0, \quad h^{a b}=\delta^{a b}, \quad h^{a \pm}=0, \quad\left(a, b=0,1,2, \ldots N^{2}\right) .
$$

Throughout this section, we always assume the basis is defined such that the metric assumes the form in (36).

\section{$5.1 \quad$ Lifted $S U(2)$}

Using the structure constants of $S U(2)$ together with (35) and (36), one can get the corresponding structure constants with four indices. Solving the gauge invariance conditions (9), we find most components of the symmetrized traces should vanish, except for

$$
\begin{aligned}
& g^{a a+}=-\frac{1}{2} g^{-++}, \quad g^{+++}, \quad d^{++++}, \quad d^{a a++}=-\frac{1}{3} d^{-+++}, \\
& d^{a a b b}=-d^{a a-+}=\frac{1}{3} d^{a a a a}=\frac{1}{2} d^{-+++}, \quad(a, b=1,2,3, \quad a \neq b)
\end{aligned}
$$


and those components obtained by reordering their indices.

\section{$5.2 \quad$ Lifted $U(2)$}

Following a similar procedure, we get the solutions to (9). Except for the components

$$
\begin{aligned}
& g^{000}, \quad g^{00+}, \quad g^{0 a a}=-g^{0-+}, \quad g^{0++}, \quad g^{a a+}=-\frac{1}{2} g^{-++}, \quad g^{+++}, \\
& d^{0000}, \quad d^{000+}, \quad d^{00++}, \quad d^{0+++}, \quad d^{++++}, \\
& d^{00 a a}=-d^{00-+}, \quad d^{0 a a+}=-\frac{1}{2} d^{0-++}, \quad d^{a a++}=-\frac{1}{3} d^{-+++}, \\
& d^{a a b b}=-d^{a a-+}=\frac{1}{3} d^{a a a a}=\frac{1}{2} d^{--++}, \quad(a, b=1,2,3, \quad a \neq b)
\end{aligned}
$$

and those obtained by reordering their indices, the other components of the symmetric tensors (4) must vanish in order to respect the gauge invariance.

In subsection 5.1 and here, some of the components can be also be fixed as before,

$$
\begin{aligned}
& g^{000}=g^{0 a a}=-\frac{i f^{3}}{4}, \quad d^{0000}=d^{00 a a}=\frac{f^{4}}{8}, \\
& d^{a a a a}=\frac{f^{4}}{8}, \quad d^{a a b b}=\frac{f^{4}}{24} . \quad(a, b=1,2,3, \quad a \neq b)
\end{aligned}
$$

\section{$5.3 \quad$ Lifted $S U(3)$}

In this example, the structure constants of the 3-algebra can be obtained by combining (16), (35) and (36). Solving the gauge invariance conditions (91), it turns out that the surviving components of the symmetrized traces are

$$
\begin{aligned}
& g^{a a+}=-\frac{1}{2} g^{-++}, \quad g^{+++}, \quad d^{++++}, \quad d^{a a++}=-\frac{1}{3} d^{-+++}, \\
& d^{a a b b}=-d^{a a-+}=\frac{1}{3} d^{a a a a}=\frac{1}{2} d^{--++}, \quad(a, b=1,2,3, \ldots, 8, \quad a \neq b)
\end{aligned}
$$

and those obtained by reordering their indices. 


\section{$5.4 \quad$ Lifted $U(3)$}

Starting with $U(3)$ algebra, repeating the above procedure, one finds the other components of (41) should vanish, except for

$$
\begin{aligned}
& g^{000}, \quad g^{00+}, \quad g^{0 a a}=-g^{0-+}, \quad g^{0++}, \quad g^{a a+}=-\frac{1}{2} g^{-++}, \quad g^{+++}, \\
& d^{0000}, \quad d^{000+}, \quad d^{00++}, \quad d^{0+++}, \quad d^{++++}, \\
& d^{00 a a}=-d^{00-+}, \quad d^{0 a a+}=-\frac{1}{2} d^{0-++}, \quad d^{a a++}=-\frac{1}{3} d^{-+++}, \\
& d^{a a b b}=-d^{a a-+}=\frac{1}{3} d^{a a a a}=\frac{1}{2} d^{-+++}, \quad(a, b=1,2,3, \ldots, 8, \quad a \neq b)
\end{aligned}
$$

and those obtained by reordering their indices.

Thanks to Gell-Mann matrices (17), we can fixed some components as

$$
\begin{aligned}
& g^{000}=g^{0 a a}=-\frac{i f^{3}}{2 \sqrt{6}}, \quad d^{0000}=d^{00 a a}=\frac{f^{4}}{12}, \\
& d^{a a a a}=\frac{f^{4}}{8}, \quad d^{a a b b}=\frac{f^{4}}{24} . \quad(a, b=1,2,3, \quad a \neq b)
\end{aligned}
$$

\subsection{Lifted $S U(N)$ and Lifted $U(N)$}

The results of the above examples are very suggestive for a generalization. They suggest that for an $S U(N)$-lifted 3-algebra with a Lorentzian metric, in order to ensure the gauge invariance (9), the non-vanishing components of symmetrized traces can only be

$$
\begin{aligned}
& g^{a a+}=-\frac{1}{2} g^{-++}, \quad g^{+++}, \quad d^{++++}, \quad d^{a a++}=-\frac{1}{3} d^{-+++}, \\
& d^{a a b b}=-d^{a a-+}=\frac{1}{3} d^{a a a a}=\frac{1}{2} d^{--++}, \quad\left(a, b=1,2,3, \ldots, N^{2}-1, \quad a \neq b\right)
\end{aligned}
$$

and those obtained by reordering their indices. For the 3-algebra extended from $U(N)$, the surviving components are

$$
\begin{aligned}
& g^{000}, \quad g^{00+}, \quad g^{0 a a}=-g^{0-+}, \quad g^{0++}, \quad g^{a a+}=-\frac{1}{2} g^{-++}, \quad g^{+++}, \\
& d^{0000}, \quad d^{000+}, \quad d^{00++}, \quad d^{0+++}, \quad d^{++++}, \\
& d^{00 a a}=-d^{00-+}, \quad d^{0 a a+}=-\frac{1}{2} d^{0-++}, \quad d^{a a++}=-\frac{1}{3} d^{-+++}, \\
& d^{a a b b}=-d^{a a-+}=\frac{1}{3} d^{a a a a}=\frac{1}{2} d^{--++}, \quad\left(a, b=1,2,3, \ldots, N^{2}-1, \quad a \neq b\right)(
\end{aligned}
$$

and those obtained by reordering their indices. 
We stress the basis of generators we employed here. When writing down the above expressions, we have assumed the basis is defined such that the metric takes the form in (36).

On the one hand, we have proved these results with $N=2,3$. On the other hand, a large class of gauge groups can be restricted to a subalgebra generated by $S U(2)$ or $S U(3)$. Hence the gauge invariance conditions presented in this subsection is not hard to understand.

Although we cannot prove the full constraints (9) be satisfied, we have checked some relations. To show a few of them, we rewrite the first equation of (9) as

$$
g^{D(A B} f_{D}^{C) F E}=0, \quad\left(A, B, \ldots=+,-, 0,1,2,3, \ldots, N^{2}-1\right)
$$

Suppose for certain $a, e, f$, we have $f^{f e}{ }_{d} \neq 0$ if and only if $d=a$. This is the case when we work in a basis such as the Chevalley basis. Then the following components of (43) lead to

$$
\begin{aligned}
(A B C E F)=(a 00 e f) & \Rightarrow g^{00-}=0, \\
(A B C E F)=(a 0+e f) & \Rightarrow g^{0 a a}+g^{0-+}=0, \\
(A B C E F)=(a++e f) & \Rightarrow 2 g^{a a+}+g^{-++}=0, \\
(A B C E F)=(e+++f) & \Rightarrow g^{a++}=0,
\end{aligned}
$$

In this way, much more components can be write down. We think the strategy depicted above and the relation between $S U(2)$ and $S U(N)$ are the key points to get a refined proof.

\subsection{Reduction to D2-branes}

The reduction of M2-branes to D2-branes is parallel to the previous section. The strategy is to choose a vacuum [11, 20, 22]

$$
<X^{-8}>=g_{Y M}
$$

with other scalar fields to be zero, and define

$$
A_{\mu}^{a-}=\frac{1}{2} A_{\mu}^{a}, \quad \frac{1}{2} \epsilon_{b c}^{a} A_{\mu}^{b c}=B_{\mu}^{a} . \quad(a, b, c \neq-)
$$

Using the equation of equation of $B_{\mu}^{a}$ (see [11, 20, 22]) at the leading order of $g_{Y M}^{-1}$, and then rescaling $X \rightarrow X / g_{Y M}$, one can get the reduced action. Since $B_{\mu}^{a}$ couples 
in Myers-Chern-Simons action (5) in the form of higher order, its equation of motion at the leading order is unchanged 4 [11, 20, 22],

$$
B_{\mu}^{a}=\frac{1}{4 g_{Y M}^{2}} \epsilon_{\mu}^{\nu \lambda} F_{\nu \lambda}^{a}-\frac{1}{2 g_{Y M}^{2}} D_{\mu} X_{a}^{8} .
$$

Neglecting terms related to goldstones and $X_{-}^{I}$, we write down the reduced action as follows

$$
\begin{aligned}
\mathcal{S}= & \int d^{3} \sigma\left(\mathcal{L}_{M C S}^{D 2}+\mathcal{L}_{H D}\right), \\
\mathcal{L}_{M C S}^{D 2}= & \frac{\lambda_{1}}{g_{Y M}^{3}} \epsilon^{\lambda \mu \nu} C_{I J K} g^{a b c} D_{\lambda} X_{a}^{I} D_{\mu} X_{b}^{J} D_{\nu} X_{c}^{K} \\
& +\frac{\lambda_{2}}{g_{Y M}^{4}} \epsilon^{\lambda \mu \nu} C_{8 I J L M N} d^{g a b c} f_{g}^{d e} X_{d}^{I} X_{e}^{J} D_{\lambda} X_{a}^{L} D_{\mu} X_{b}^{M} D_{\nu} X_{c}^{N}, \\
\mathcal{L}_{H D}= & -\frac{3 \lambda_{1}}{g_{Y M}^{3}} C_{8 I J} g^{a b c} F_{a}^{\mu \nu} D_{\mu} X_{b}^{I} D_{\nu} X_{c}^{J},
\end{aligned}
$$

with $I, J, \ldots=1,2,3, \ldots, 7$ and $a, b, c \ldots=0,1,2, \ldots, N^{2}-1$. Higher order terms with respect to $g_{Y M}^{-1}$ are neglected because the reduction is done in the limit $g_{Y M} \rightarrow \infty$. We also removed the ghost fields by setting $X_{+}^{I}=$ constant [22].

Notice here the second term of $\mathcal{L}_{M C S}^{D 2}$ takes a different sign from that in (32). This is because structure constants $f_{g}^{4 d e}$ in $\mathcal{A}_{4}$ and $f_{g}^{+d e}$ in the present 3-algebras relate to $f^{d e}$ of Lie 2-algebras in different ways,

$$
f_{g}^{4 d e}=-f_{g}^{d e}, \quad f_{g}^{+d e}=f_{g}^{d e} .
$$

The surviving terms contain Myers-Chern-Simons terms for D2-branes. These are exactly what we expected. Once again we get a high-dimensional term $\mathcal{L}_{H D}$. This term is proportional to $g_{Y M}^{-3}$, which is of the leading order in (48).

\section{On Cubic Matrices}

In subsection 4.3, we saw that under a simple definition of trace (28), the cubic matrix representation (29) fails to reproduce the required non-vanishing components. Some comments are needed here.

First, one can make use of the representation (29) and rule (30) to work out the symmetrized triple product

$$
\left\{T^{a}, T^{b}, T^{c}\right\}_{i j k}=6\left(T^{(a}, T^{b}, T^{c)}\right)_{i j k}=-i \sum_{d}\left|\epsilon^{a b c d}\right| \epsilon^{d i j k}\left(T^{d}\right)_{i j k} .
$$

\footnotetext{
${ }^{4}$ To make the convention of notations in accordance with (48), when writing down (46) and (47) we have already rescaled $A \rightarrow A / 2$ and $X \rightarrow X / g_{Y M}$ as did in [1].
} 
This expression and the results in section 4 would be helpful for exploring a possibly better definition of trace instead of (28).

Second, the definition (30) of the triple product is not unique in principle. Here are two alternative schemes:

$$
\begin{aligned}
& (A, B, C)_{l m n}=\sum_{i, j, k} A_{l i j} B_{m j k} C_{n k i}, \\
& (A, B, C)_{l m n}=\sum_{i, j} A_{l i j} B_{m i j} C_{n i j},
\end{aligned}
$$

which are easy to be generalized for multiple products of cubic matrices. Their drawback is: if we take the structure constants in (18) as a generalized "adjoint representation" 5 of $\mathcal{A}_{4}$ algebra, then only the anti-symmetrized product based on (30) can recover $\mathcal{A}_{4}$ algebraic relation (18). But they also have their virtues. Suppose a certain generator $T^{a}$ in the cubic representation satisfying

$$
\left(T^{a}\right)_{i j k}=\left(T^{a}\right)_{j k i}=\left(T^{a}\right)_{k i j}, \quad\left(T^{a}\right)_{i j j}=0 .
$$

When (30) is applied, this will inevitably lead to $g^{0 a a}=0$, which is at odds with (26). In contrast, neither (51) nor (52) suffers for such a problem.

The above problem also inspires us to search for cubic matrix representations $\left(T^{a}\right)_{i j j} \neq 0$ breaking the condition (53). This is the third possibility one may try to translate the 3 -algebraic rules into the language of cubic matrices.

\section{Conclusion}

In this paper, we studied Myers-Chern-Simons action for multiple M2-branes. We wrote down the gauge invariance conditions and solved them in some concrete examples. One example is the gauge theory based on $\mathcal{A}_{4}$ algebra. The other class of examples are non-Abelian gauge theories by lifting $S U(N)$ or $U(N)$ to 3-algebras with a Lorentzian signature. The reduction of M2-branes to D2-branes puts addtional constraints on the Myers-Chern-Simons action.

We studied simpler examples first and checked a few relations for the lifted $U(N)$, In the case of $\mathcal{A}_{4}$, we found all of the components can be fixed up to an overall normalization. For 3-algebras with a Lorentzian signature, only some components can be fixed.

\footnotetext{
${ }^{5}$ The cubic matrices (29) play a similar role.
} 
We also offered some tentative thoughts on cubic matrices as the representations of 3-algebras. In spite of notorious difficulty, this is a direction deserving to follow up in the future. We plan to study some physical consequences of the terms introduced in this paper, and their supersymmetric generalization in a future paper.

Acknowledgement: We would like to thank Yushu Song for substantial discussions and Qin-Yan Tan for kind help in programme. This work was supported by grants of CNSF and grants of USTC.

\section{References}

[1] J. Polchinski, Phys. Rev. Lett. 75, 4724 (1995) [arXiv:hep-th/9510017].

[2] M. Li, Nucl. Phys. B 460, 351 (1996) arXiv:hep-th/9510161.

[3] M. R. Douglas, arXiv:hep-th/9512077.

[4] A. A. Tseytlin, Nucl. Phys. B 501, 41 (1997) arXiv:hep-th/9701125].

[5] R. C. Myers, JHEP 9912, 022 (1999) arXiv:hep-th/9910053.

[6] J. Bagger and N. Lambert, Phys. Rev. D 75, 045020 (2007) arXiv:hep-th/0611108.

[7] J. Bagger and N. Lambert, Phys. Rev. D 77, 065008 (2008) arXiv:0711.0955 [hep-th]].

[8] J. Bagger and N. Lambert, JHEP 0802, 105 (2008) arXiv:0712.3738 [hep-th]].

[9] A. Gustavsson, arXiv:0709.1260 [hep-th].

[10] A. Gustavsson, JHEP 0804, 083 (2008) arXiv:0802.3456 [hep-th]].

[11] S. Mukhi and C. Papageorgakis, arXiv:0803.3218 [hep-th].

[12] U. Gran, B. E. W. Nilsson and C. Petersson, arXiv:0804.1784 [hep-th].

[13] P. M. Ho, R. C. Hou and Y. Matsuo, arXiv:0804.2110 [hep-th].

[14] P. M. Ho and Y. Matsuo, arXiv:0804.3629 [hep-th].

[15] P. M. Ho, Y. Imamura, Y. Matsuo and S. Shiba, arXiv:0805.2898 [hep-th].

[16] G. Papadopoulos, arXiv:0804.2662 [hep-th]. 
[17] J. P. Gauntlett and J. B. Gutowski, arXiv:0804.3078 [hep-th].

[18] M. A. Bandres, A. E. Lipstein and J. H. Schwarz, arXiv:0803.3242 [hep-th].

[19] H. Awata, M. Li, D. Minic and T. Yoneya, JHEP 0102, 013 (2001) arXiv:hep-th/9906248.

[20] J. Gomis, G. Milanesi and J. G. Russo, arXiv:0805.1012 [hep-th].

[21] S. Benvenuti, D. Rodriguez-Gomez, E. Tonni and H. Verlinde, arXiv:0805.1087 [hep-th].

[22] P. M. Ho, Y. Imamura and Y. Matsuo, arXiv:0805.1202 [hep-th].

[23] A. Morozov, arXiv:0804.0913 [hep-th].

[24] A. Morozov, arXiv:0805.1703 [hep-th].

[25] N. Lambert and D. Tong, arXiv:0804.1114 [hep-th].

[26] J. Distler, S. Mukhi, C. Papageorgakis and M. Van Raamsdonk, arXiv:0804.1256 [hep-th].

[27] H. Fuji, S. Terashima and M. Yamazaki, arXiv:0805.1997 [hep-th].

[28] D. S. Berman, L. C. Tadrowski and D. C. Thompson, arXiv:0803.3611 [hep-th].

[29] M. Van Raamsdonk, arXiv:0803.3803 [hep-th].

[30] J. Gomis, A. J. Salim and F. Passerini, arXiv:0804.2186 [hep-th].

[31] E. A. Bergshoeff, M. de Roo and O. Hohm, arXiv:0804.2201 [hep-th].

[32] K. Hosomichi, K. M. Lee and S. Lee, arXiv:0804.2519 [hep-th].

[33] G. Papadopoulos, arXiv:0804.3567 [hep-th].

[34] Y. Honma, S. Iso, Y. Sumitomo and S. Zhang, arXiv:0805.1895 [hep-th].

[35] C. Krishnan and C. Maccaferri, arXiv:0805.3125 [hep-th].

[36] Y. Song, arXiv:0805.3193 [hep-th].

[37] I. Jeon, J. Kim, N. Kim, S. W. Kim and J. H. Park, arXiv:0805.3236 [hep-th].

[38] P. M. Ho and Y. Matsuo, Gen. Rel. Grav. 39, 913 (2007) arXiv:hep-th/0701130. 
[39] Y. Kawamura, Prog. Theor. Phys. 110, 579 (2003) arXiv:hep-th/0304149].

[40] Y. Kawamura, Prog. Theor. Phys. 114, 669 (2005) [arXiv:hep-th/0504017]. 